Karl Traugott Goldbach (Kassel)

\title{
Louis Spohr und der Kinnhalter
}

Auch Geiger, die noch nie ein Werk von Louis Spohr gespielt haben, wissen häufig: Das war doch der, der die Kinnstütze erfunden haben soll. 1833 ging Spohr mit dieser von ihm noch „Geigenhalter“ genannten Erfindung bei der Veröffentlichung seiner Violinschule an die Öffentlichkeit (vgl. Abb. 1):

„Am unteren Theil des Fig. I abgebildeten Instruments, über dem Saitenhalter befindet sich noch eine, von mir erfundene Vorrichtung, der Geigenhalter (1.) genannt, die nach mehr als zehnjährigem Gebrauch bey mir, meinen zahlreichen Schülern und vielen andern Geigern ihren Nutzen bewährt hat und von der ich daher hier wohl einige Worte sagen muss." 1

Leider gibt Spohr in dieser Beschreibung keine genauen Maße an, weshalb eine Rekonstruktion seines Geigenhalters schwierig ist:

„Der Geigenhalter wird von Ebenholz in der, auf der Zeichnung, (Fig. II, 1.2.3.) von verschiedenen Seiten, gegebenen Form verfertigt und mit einem Zapfen (a.) in der Öffnung, die früher der Knopf ausfüllte, befestigt. Die Saite, an welcher der Saitenhalter hängt, läuft um den Zapfen in der, für sie bestimmten Vertiefung. (b.) Der Knoten (c.) wird über dem Saitenhalter geknüpft, doch so, dass er den Geigenhalter nicht berührt. Für den kleinen Sattel, auf welchem die Saite ruht, so wie für den Rand der Geige wird ebenfalls eine Vertiefung (d.) eingeschnitten, damit der Geigenhalter sich dicht an die Zargen anschliessen kann. Die obere Scheibe wird in der Mitte etwas vertieft, (c.) damit das Kinn um so fester und bequemer darauf ruhe. Der Zapfen muss sehr genau in die Öffnung passen, damit er durch den starken Zug der Saite nicht herausgehoben werden kann. " ${ }^{2}$

Ein originaler Kinnhalter Spohrs ist derzeit nicht nachweisbar. Ein früher im Musikinstrumenten-Museum Berlin nachgewiesener „Kinnhalter, angeblich der von Louis Spohr“3 ist offensichtlich ein Kriegsverlust. ${ }^{4}$ Ein Geigenhalter nach Spohr im Bestand des Danish Music Museum ist nur an Spohr angelehnt. ${ }^{5}$ Die leicht asymmetrische Form zeigt deutlich, dass hier das Kinn nicht, wie von Spohr gefordert, direkt über dem Saitenhalter aufliegt, sondern links vom Saitenhalter, wie heute meist üblich; außerdem ist dieser Geigenhalter nicht wie bei Spohr aus Ebenholz, sondern aus Mahagoni gefertigt (vgl. Abb. 2a und 2b). ${ }^{6}$

Mittlerweile ist die Verwendung des Kinnhalters für die meisten Geiger selbstverständlich geworden. Dabei gelten immer noch die gleichen Gründe, die Spohr selbst für seine Erfindung gab:

„Die neuere Spielart, bey der die linke Hand so oft die Lage wechselt, macht es durchaus nöthig, dass die Violine mit dem Kinn festgehalten werde. Diess auf ungezwungene Weise und ohne dass der Kopf

1 Louis Spohr, Violinschule, Wien 1833, S. 8.

2 Ebd., S. 9.

3 Oskar Fleischer, Führer durch die Sammlung alter Musikinstrumente, Berlin 1892, S. 95.

4 Freundliche Mitteilung des Musikinstrumentenmuseums Berlin.

5 Vgl. Angul Hammerich, Das Musikhistorische Museum zu Kopenhagen. Beschreibender Katalog, Kopenhagen 1911, S. 100.

6 Abb. 2b zeigt vielleicht noch nachvollziehbarer als die Zeichnungen in Spohrs Violinschule wie der Geigenhalter an der Geige angebracht wird. 


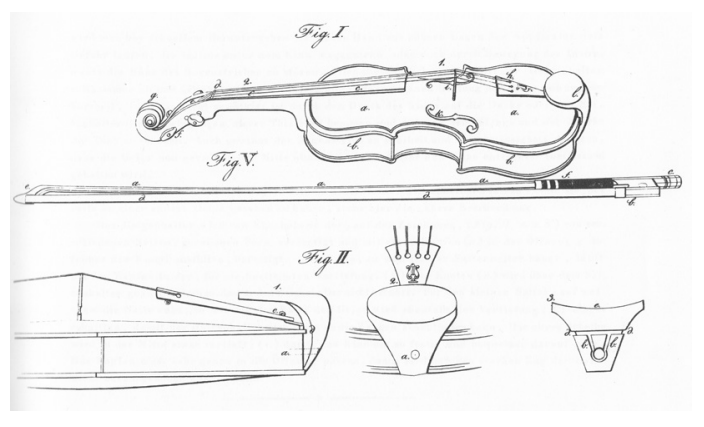
Abbildung 1: Spohr, Violinschule, 1tes Blatt (nach S. 8).
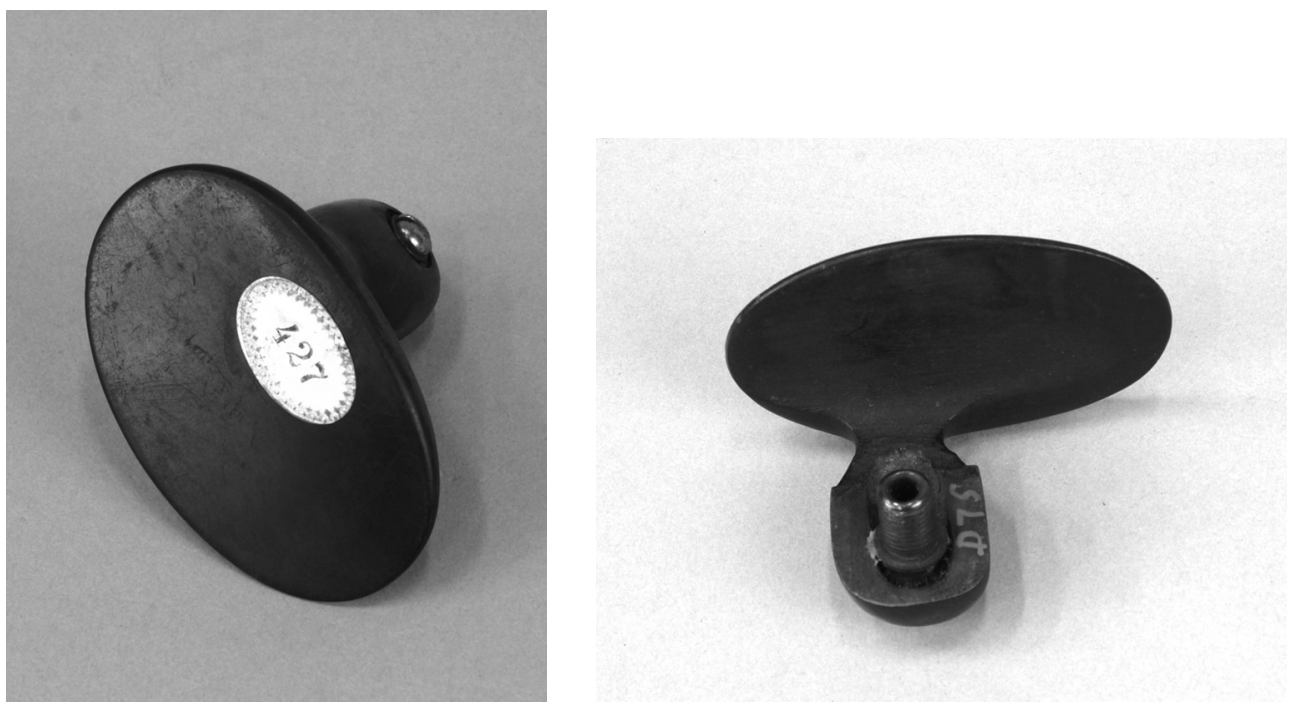

Abbildungen 2a und 2b: An Spohrs Beschreibungen angelehnter Geigenhalter, DK-Km MMCCS No. D 74, Abdruck mit freundlicher Genehmigung durch The Danish Music Museum - Musikhistorisk Museum \& The Carl Claudius Collection.

niedergebückt werde, zu thun, hat seine grossen Schwierigkeiten, man mag das Kinn auf die rechte oder linke Seite des Saitenhalters oder gar auf diesen selbst legen. Auch wird man bey schnellem Heruntergehen der linken Hand aus höhern Lagen der Applicatur stets Gefahr laufen, die Violine unter dem Kinn wegzuziehn oder doch durch Bewegung des Instruments die Ruhe des Bogenstrichs zu stören. Allen diesen Übelständen hilft der Geigenhalter vollkommen ab und gewährt neben der festen und ungezwungenen Haltung der Geige auch noch den Vortheil, dass man nicht genöthigt ist durch den Druck des Kinns auf die Decke oder den Saitenhalter die Schwingungen dieser Theile zu hemmen und dadurch dem Klange und der Stärke des Tons zu schaden. Auch gewinnt der Bogenstrich an Freiheit und Regelmässigkeit dadurch, dass die Geige nun gerade in der Mitte über dem Saitenhalter und etwas entfernter vom Gesicht gehalten wird. "7

Jedem Geiger der Zeit ist das Problem des Wechsels aus hohen in tiefere Lagen bewusst. Daher erstaunt eine bewundernde Einschätzung des Kinnhalters im Anschluss an eine Rezension der Violinschule kaum:

7 Spohr, Violinschule, S. 8f. 
„Der Vortheil einer solchen Einrichtung ist so ganz und gar in die Augen fallend, dass man sich eigentlich nur wundern mögte, dass es erst eines Spohr bedurfte, sie zu erfinden, indess man denken sollte, schon Hr. Vater Adam im Paradiese, wenn er anders Violine spielte, hätte wohl schon so klug sein können, seine Stradivarius nicht ohne Geigenhalter halten zu wollen. “8

Obwohl dieser Autor offensichtlich ein Anhänger der neuen Erfindung ist, kennt er aus Diskussionen wohl auch skeptische Einschätzungen. Zumindest setzt er sich in einem weiteren Abschnitt gleich mit einem Einwand zum Geigenhalter auseinander:

„Dass dem, sich einmal an diese Vorrichtung gewöhnt habenden Spieler, dieselbe zum Gewohnheitsbedürfnisse wird, indess es ja doch unter vielen tausenden unserer jetzt existierenden Geigen kaum eine mit einem Geigenhalter versehene finden, und daher überall, wo er nicht sein eigenes gewöhntes Instrument bei sich hat, sich in Verlegenheit finden wird, - das alles ist nur halbwahr! - denn für's Erste sind wir unbesehens überzeugt, dass der durch seinen Geigenhalter seit vielen Jahren verwöhnte Hr. Spohr auch auf Violinen ohne Geigenhalter doch noch ganz artig geigen wird; - und was gerade das grosse Bravour- und Concertspiel angeht, - so pflegt ja dazu doch jeder Virtuose sich nur seines eigenen Instrumentes zu bedienen; - gar nicht einmal zu erwähnen, dass es es eben keine herkulische Aufgabe sein mögte, einen Geigenhalter augenblicklich an jede Violine zu befestigen. “9

So „augenblicklich“ war das Befestigen freilich nicht zu bewerkstelligen. Wie aus Spohrs oben wiedergegebener Beschreibung ersichtlich, wird mit dem Spohr'schen Geigenhalter anstelle des Sattelknopfs der Saitenhalter an der Violine befestigt; vor allem muss dabei „der Zapfen [...] sehr genau in die Öffnung passen, damit er durch den starken Zug der Saite nicht herausgehoben werden kann". Abgesehen davon, dass nach dem Befestigen des Geigenhalters so immer die Saiten neu gespannt werden müssten, ist ein Geigenhalter auch eine Maßanfertigung für die jeweilige Geige. Dies erklärt Spohr 1838 ausdrücklich in einem Brief an seinen Vetter Friedrich:

„Der hiesige Instrumentenmacher ist nicht im Stande den verlangten Geigenhalter zu verfertigen ohne die Geige zu haben, für welche er bestimmt ist, da er an diese auf das genaueste angepaßt und befestigt werden muß. Sollte daher der Geigenhalter nicht in Braunschweig gemachet werden können, so müßte sich Herr Hohenstock entschließen die Geige hieher zu schicken. Binnen höchstens 8 Tagen würde er sie zurückerhalten. Der Instrumentenmacher heißt Schonger und wohnt in der Königsstraße im Bachfeld'schen Hause. Er ist sehr geschickt und man kann ihm unbedenklich jedes Instrument anvertrauen. $[\ldots]^{\text {“10 }}$

Spohr schlägt nicht etwa vor, ein beliebiger anderer Geigenbauer könne den Geigenhalter anfertigen, sondern er empfiehlt den Kasseler Geigenbauer Joseph Schonger. Möglicherweise wollte Spohr so Schonger einen Auftrag verschaffen, der erst seit einem knappen Jahr in Kassel tätig war. Dies geht aus einem Brief hervor, den Spohr 1837 an den Instrumentenbauer Wilhelm Ehrlich ${ }^{11}$ schrieb, der sich in Kassel niederlassen wollte und Spohr dazu um Unterstützung gebeten hatte:

8 [Gottfried Weber?], „Nachschrift der Redaction“, in: Cecilia 15 (1833), S. 282-292, hier S. 285.

9 Ebd., S. $285 f$.

10 Louis Spohr, Brief an Friedrich Spohr in Braunschweig, Kassel 29. August 1838, D-Ksp Sp. ep. 1.3 $<$ Spohr, Friedrich 18380829>.

11 Lütgendorff gibt für Ehrlich als Lebensdaten an: „Geb. um 1820, † 4. März 1887“ (Willibald Leo von Lütgendorff, Die Geigen- und Lautenmacher vom Mittelalter bis zur Gegenwart, Frankfurt a. M. 1904, S. 159; in der im Faksimile Tutzing 1975 leichter greifbaren 6. Aufl. Bd. 2, S. 120). Spohrs Brief setzt m. E. voraus, dass Ehrlich 1837 bereits seine Meisterprüfung hinter sich hat. Der Termin seiner Geburt wäre folglich deutlich früher anzusetzen. 
„Es ist mir leid, Ihnen eine unangenehme Nachricht mittheilen zu müssen. Ein Sohn des Instrumentenmachers Schonger in Erfurt, der schon seit mehreren Jahren auf 4-6 Monathe hieher kam, um hier zu repariren, ist auf dieselbe Idee gekommen wie Sie, nämlich sich hier zu besetzen. Wie ich Ihnen auf Ihre Anfrage antwortete, war er bereits hier und hatte auch schon Schritte gethan um die Concession zu erhalten, wovon ich jedoch nichts wußte. Nun hat er sie erhalten und wird auf Neujahr hieherziehen. $\mathrm{Da}$ er sich hier in seinen Arbeiten als ein sehr geschickter Mann gezeigt hat, so konnte ich ihm nicht entgegen seyn. Ich muß daher bedauern unter diesen Umständen für Ihren Wunsch nichts thun zu können."12

Der Exkurs zum Geigenbau in Kassel mag auf den ersten Blick vom Thema dieses Texts ablenken. Doch eine biographische Anmerkung zu Schonger zeigt, dass Spohr als Erfinder des Geigenhalters in Vergessenheit geriet; zumindest scheint Lütgendorff 1904 in seinem Verzeichnis der Geigen- und Lautenmacher nicht Spohr, sondern Schonger die Erfindung zuzuschreiben: „Er [Schonger] erfand auch einen jetzt vielverbreiteten Kinnhalter“. ${ }^{13}$ Aus der knappen Bemerkung geht freilich nicht zwingend hervor, dass Lütgendorff Schonger für den Erfinder des Kinnhalter hält; denkbar wäre auch die Lesart, Schonger habe eine bestimmte Form des Kinnhalters entwickelt. Dafür könnte auch die Formulierung „einen jetzt vielverbreiteten Kinnhalter“ sprechen. Dann wäre Schongers Erfindung ein bestimmter Kinnhalter, wobei das „vielverbreitet“ beinhalten würde, dass es noch sehr viele Geiger gab, die einen anderen Kinnhalter verwendeten. Zugleich könnte „vielverbreitet“ aber auch bedeuten, dass viele Geiger einen Kinnhalter verwendeten, andere jedoch darauf verzichteten. Dass um diese Zeit sowohl die Form des Geigenhalters, wie Spohr sie vorsah, als auch die Urheberschaft Spohrs nicht mehr allgemein bekannt waren, geht aus einem knappen Text von Benno Hirsch aus dem Jahre 1909 hervor, der sich mit Spohrs Geigenhalter auseinandersetzt, um ein eigenes Patent zu verteidigen:

„Durch eine Zuschrift an ein Münchener Blatt, durch welche der Rechtsbestand eines mir erteilten Patents in Frage gestellt wurde, war es mir vergönnt, Spohr als den eigentlichen Begründer des Geigenhalters kennen zu lernen. Erst durch diese Zuschrift wurde ich auf die Spohrsche Violinschule von 1832 aufmerksam gemacht, welche ich erst nach langem vergeblichem Suchen in der Staatsbibliothek und in den Musikalienhandlungen, endlich in der Bibliothek der Akademie der Tonkunst, hier [München], auffand. Es scheint dies also ein äußerst seltenes Werk zu sein." ${ }^{14}$

Der Widerspruch gegen Hirschs Patent entzündete sich daran, dass sein Geigenhalter wie der Spohrs im Gegensatz zu den zu dieser Zeit üblichen nicht links vom Saitenhalter, sondern darüber befestigt war. Der weitere Verlauf dieser Auseinandersetzungen würde den Rahmen dieses Texts sprengen. Bemerkenswert für unseren Zusammenhang ist, dass

12 Louis Spohr, Brief an Wilhelm Ehrlich in Dresden, Kassel 21. September 1837, D-Ksp Sp. ep. 1.6 <Ehrlich, Wilhelm 18370921>. - Lütgendorff behauptet: „Nachdem er ausgelernt hatte, blieb er [Schonger, Anm. K.T.G.] noch bis 1838 in Erfurt und kam im Mai des genannten Jahres besuchsweise nach Kassel, wo ihn Spohr veranlaßte seinen bleibenden Wohnsitz aufzuschlagen" (Geigen- und Lautenmacher, S. 577 (6. Aufl., Bd. 2, S. 452)). Spohrs eigene Angabe im zitierten Brief, er habe nicht gewusst, dass Schonger sich in Kassel niederlassen wolle, widerspricht dieser Darstellung. Mangels weiterer Quellen lässt sich nicht feststellen, welche der beiden Versionen zutrifft. Die Angabe, Schonger sei zunächst besuchsweise in Kassel gewesen, stimmt in beiden Texten überein, der zeitnähere und in diesem Punkt daher glaubwürdigere Spohr-Brief datiert die Geschehnisse allerdings ein Jahr früher.

13 Lütgendorff, Geigen- und Lautenmacher, S. 577 (6. Aufl., Bd. 2, S. 452).

14 Benno Hirsch, „Louis Spohr als Erfinder des Geigenhalters“, in: Allgemeine Musik-Zeitung 35 (1908), S. 652 . 
Hirsch den Geigenhalter als „absolut unentbehrliches Zubehör für die Geige bezeichnet“ und gleichzeitig feststellt, Spohr sei als Erfinder in Vergessenheit geraten. ${ }^{15}$

Fünf Jahrzehnte früher, 1869, weist Joseph von Wasiliewski, der selbst beim SpohrSchüler Ferdinand David studiert hatte, noch auf Spohr als Erfinder hin, erkennt den Kinnhalter jedoch gerade nicht als absolut unentbehrlich an:

„Eine freilich nur nebensächliche von Spohr eingeführte Neuerung, der tellerförmige, über dem Saitenhalter angebrachte Kinnhalter nämlich, welcher hauptsächlich darauf berechnet war, dem Kinn eine feste Stütze zu geben, ohne einen Druck auf die Oberdecke der Violine auszuüben, fand nicht Eingang, da sie tatsächlich keinen Vortheil gewährte."16

Wasiliewskis Lehrer David erwähnt in seiner Violinschule den Geigenhalter in Bezug auf die Haltung des Instruments nicht, wenn er die Haltung der Violine beschreibt: „Das Kinn ruht dicht am Saitenhalter auf der linken Seite, der Kopf etwas nach derselben Seite geneigt." ${ }^{77}$ Die von Spohr durch den Geigenhalter beabsichtigte stabile Haltung der Geige zwischen Schulter und Kinn erreicht David durch eine Vorform der Schulterstütze:

„Die neuere Spielart, welche einen häufigen Wechsel der Lagen erfordert, bedingt eine feste Lage der Violine, welche man am bequemsten erreicht, indem man ein Tuch oder ein kleines Kissen zwischen die Violine und die linke Schulter legt. Letztere braucht dann nicht zu weit heraufgezogen zu werden und die Haltung ist ungezwungener." 18

Ein weiterer Spohr-Schüler, Hubert Ries, der sein Lehrwerk ausdrücklich als Ergänzung von Spohrs Violinschule für den Anfangsunterricht konzipiert ${ }^{19}$, geht offensichtlich genausowenig vom Geigenhalter aus: „Viertens, lege man das Kinn sanft auf die linke Seite der Violine neben den Saitenhalter, und neige dabei den Kopf ein wenig nach links. “20

Wasiliewskis Text verweist - wohl unabsichtlich - auf den Ausgangspunkt von Spohrs Überlegungen bei der Entwicklung des Geigenhalters. Tatsächlich ging es Spohr zunächst nicht darum, dem Kinn eine sichere Stütze zu verleihen, sondern den Druck des Kinns auf die Oberdecke zu vermeiden. Dabei war der Geigenhalter zunächst auch nur das Nebenprodukt eines von Spohr weiterentwickelten Saitenhalters. Spohr fügt einem Bericht über das Londoner Musikleben etwas unmotiviert an:

„Nun zum Schluss noch einige Worte über eine Verbesserung an der Geige, die ich von den neuerfundenen Geigen des Hrn. Chanot auf mein Instrument übertragen habe. Sie besteht darin, dass ich den Saitenfessel so sehr habe verkürzen lassen, dass die 4 Saiten diesseits des Stegs jetzt folgende Töne geben:

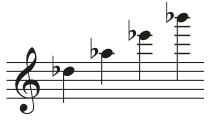

Der Gewinn ist erstens eine leichtere Ansprache des Instruments überhaupt, und dann zweytens das Mitklingen dieser vier Töne, wodurch nun die bisher stumpfen Tonarten der Geige fast eben so brillant geworden sind, als die, bey deren Intervallen die bloßen Saiten mitklingen. Es ist mir seit dieser Veränderung oft geschehen, dass man, wenn ich in Des dur präludierte, es für D dur nahm. Die Vorrichtungen, die ich mit Hülfe eines geschickten Instrumentenmachers in Lille, des Hrn. Lannoye, dazu

15 Ebd.

16 Joseph Wilhelm von Wasiliewski, Die Violine und ihre Meister, Leipzig 1869, S. 337.

17 Ferdinand David, Violinschule, Leipzig [1863], Bd. 1, S. 5.

18 Ebd.

19 Hubert Ries, Violinschule für den ersten Unterricht. Nebst 106 kleinen Duetten und zweckmäßigen Uebungsstücken, Leipzig 1840, Bd. 1, S. 4.

20 Ebd., S. 8. 
ersann, bestehen erstens in der Weise, wie die Saiten unterhalb des Saitenfessels befestigt, zweytens in einem Schilde, der sie, ohne sie zu berühren, so weit bedeckt, dass das Kinn beym Geigehalten nicht mehr anstoßen kann, und drittens in einem Sattel unterhalb des Saitenfessels, auf dem die 4 Saiten ruhen; und mit dem sie rein abgestimmt werden. Die Londoner Geigenmacher, die für die dortigen Künstler und Dilettanten viele solcher Saitenhalter verfertigten, haben noch einige Verbesserungen angebracht, die sich aber ohne Zeichnungen nicht deutlich machen lassen. Sollte einer meiner Landesleute einen solchen kleinen Saitenhalter zu besitzen wünschen, so werde ich gern irgend einem deutschen Instrumentenmacher ein Modell geben, nach welchem er sie verfertigen kann. “21

Etwas ausführlicher beschrieb Spohr die Konstruktion unmittelbar vor seinem LondonAufenthalt in einem Brief an seinen Freund Wilhelm Speyer:

„Die erste Idee zu dieser Veränderung gab mir die Geige nach der neuen Erfindung die ich Ihnen im vorigen Br. beschrieben habe. Sie besteht darin daß ich mir habe einen neuen ganz kurzen Saitenfessel machen lassen, so daß die Saiten ganz nah am Knopf, unter dem Kinn, befestigt sind. Damit man sie nicht mit dem Kinn berühre, habe ich eine Art von Schild darüber machen lassen, der aber die Saiten nicht berührt und sie in ihren Schwingungen nicht stört. Damit Sie mich besser verstehen will ich die Geige hieher zeichnen. Die Saiten laufen unter dem Saitenfessel fast so weit die Punkte reichen und sind dann unterwärts befestigt, so daß der Saitenfessel oben ganz schlicht(?) ist. Damit man besser dazu kann wenn eine reißt, habe ich ein Loch bohren lassen wodurch man die Knoten der beyden mittleren sieht. Dies Loch ist aber durch einen mit Perlmutt verzierten Knopf geschlossen der nach Belieben herausgenommen werden kann. Es ist überdies unter dem Saitenfessel ein kleiner Steg auf welchem die Saiten ruhen und wodurch sie ganz genau in folgende Quinten gestimmt sind. [hier folgt das gleiche Notenbeisp. wie in der $A M Z$ ] Diese vier stumpfesten Töne der Geige finden nun diesseits des Stegs einen Mitklang, wodurch auf meiner Geige Desdur beynah so brillant klingt wie Ddur auf andern. Außerdem hat die Geige überhaupt sehr an Klang und Stärke gewonnen und noch ein großer Vortheil ist, daß alle Saiten die ich aufziehe,

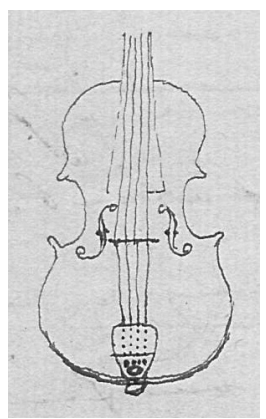
sie möge seyn wie sie wollen, quintenrein sind, eine Sache, die ich noch nicht recht zu erklären weiß, die aber unbezweifelt und für den Spieler von unendlichem Vortheile. Da ich sehr wünsche, daß diese Veränderung vor meiner Rückkehr nach Deutschland nicht nachgemacht werde, so bitte ich Sie, diese Beschreibung niemand zu zeigen der Gebrauch davon machen könnte. “22

Aus der von Spohr erwähnten Zeichnung wird die Konstruktion etwas klarer (vgl. Abb. 3). Dort wo das „Schild“ die Saiten überragt, sind diese in der Zeichnung nur gestrichelt dargestellt. Die Vorrichtung zum Stimmen der kürzeren Saitenenden ist hier jedoch nicht zu erkennen. Anscheinend handelt es sich bei dem „Schild“ über dem „Saitenfessel“

Abbildung 3: Zeichnung Spohrs in seinem Brief an Wilhelm Speyer, 21. Februar 1820, D-B 55 Nachl. 76,13, Abdruck mit freundlicher Genehmigung durch die Staatsbibliothek Berlin Preußischer Kulturbesitz - Musikabteilung mit Mendelssohn-Archiv.

21 Louis Spohr, „Musikalische Notizen gesammelt von Louis Spohr während seines Aufenthalts in London vom Ende Februar bis Ende July 1820“, in: Allgemeine musikalische Zeitung 22 (1820), Sp. 521-530, hier Sp. 530.

22 Louis Spohr, Brief an Wilhelm Speyer in Frankfurt a. M., Lille 23. Februar 1820, D-B 55 Nachl. 76,13. - In der vorliegenden Teiledition des Spohr-Speyer-Briefwechsels ist die hier zitierte Passage weggekürzt (Eduard Speyer, Wilhelm Speyer der Liederkomponist 1790-1878. Sein Leben und Verkehr dargestellt von seinem jüngsten Sohne, München 1925, S. 42f.). 
bereits um den Geigenhalter. ${ }^{23}$ Offensichtlich erkannte Spohr dessen Vorteil aber erst in der folgenden Zeit.

Dafür faszinierte der Saitenhalter kurzfristig die Musikwelt. D-Dur klingt auf der Geige auch deshalb so brillant, weil die gerade nicht verwendeten leeren Saiten mitschwingen und dem Klang so zusätzliche Obertöne zufügen. Durch das Einstimmen der Saitenenden jenseits des Saitenhalters auf Obertöne zu Des-Dur glaubt Spohr, einen ähnlichen Effekt nun auch für schwerer ansprechende B-Tonarten zu erzeugen. Spohr berichtet selbst über das Interesse, welches er in London damit auslöste. Auf zwei Quellen weist Clive Brown hin. ${ }^{24}$ Zum einen auf einen weiteren Brief Spohrs an Speyer, in dem er nochmals das Interesse an seiner Erfindung unterstreicht: „Meine Veränderung an der Geige bewährt sich als vortrefflich; die hiesigen Künstler fangen schon an, sie nachzumachen“. 25 Die zweite Quelle gibt jedoch einen Hinweis, dass hier eine andere als von Spohr selbst gesehene Qualität empfunden wurde:

„There is also a pecularity in the fitting up of his violin, the tail-piece being considerably shorter than those in general use. This construction is said to give a quicker return of the string from the fingerboard, and to confer greater facility in execution. " 26

Der kürzere Saitenhalter ergibt sich daraus, dass Spohr - wie oben beschrieben - auch die kurzen Enden der Saiten einstimmt. Der hier in diesem Zitat behauptete Vorteil ist jedoch nicht der von Spohr bezweckte ausgewogenere Klang, sondern eine durch schnellere Rückkehr der Saite vom Griffbrett erreichte leichtere Spielbarkeit.

Das „Interesse, welches viele an dem verbesserten Saitenfessel nahmen (worüber Spohr selbst in seinen musikalischen Notizen aus London Auskunft gibt), dessen Wirkung nun erprobt werden sollte“, blieb bei einem kurze Zeit später erfolgten Frankfurter Konzert kaum hinter den Erwartungen an das Konzert selbst zurück. ${ }^{27}$ Die vom Publikum empfundene Verbesserung von Spohrs Ton führt der Kritiker dann auch auf den Saitenhalter zurück: „Allerdings mag die oben erwähnte Verbesserung des Saitenhalters auch dazu beytragen; denn es ist nicht zu läugnen, dass seine Geige an Klang, Stärke (besonders auf dem $G$ ) und Ausgeben der Töne sehr gewonnen hat." ${ }^{28}$ Da die Verbesserung des $G$ durch den Saitenhalter von Spohr gerade nicht angestrebt war, sei dahingestellt, ob die Frankfurter Hörer wirklich eine Klangverbesserung durch den neuen Saitenhalter wahrnehmen konnten.

Deutsche Geigenbauer gingen auf Spohrs Angebot ein, Saitenhalter nach einem zur Verfügung gestellten Modell zu fertigen. Der Gothaer Instrumentenmacher Johann Gott-

23 Den frühesten mir bekannten Hinweis auf diesen Zusammenhang gab Folker Göthel: „Dort wird auch erstmals der von Spohr erfundene Geigenhalter erwähnt“ („Anmerkungen“, in: Louis Spohr, Lebenserinnerungen, hrsg. von Folker Göthel, Tutzing 1968, Bd. 2, S. 235, Anm. 22). Göthel stellt allerdings nicht fest, dass es sich hier um ein Nebenprodukt zum Saitenhalter handelt, sondern bemerkt nur: „Von diesem Instrument [von Chanot] übernahm Spohr die Praxis des Abstimmens der Saiten zwischen Steg und Saitenhalter [...]" (ebd.).

24 Clive Brown, Louis Spohr. A critical Biography, Cambridge 1984, S. 131; deutsche Übersetzung: ders., Louis Spohr. Eine kritische Biographie, übersetzt von Wolfram Boder, Kassel 2009, S. 157.

25 Louis Spohr, Brief an Wilhelm Speyer in Frankfurt a. M., London 27. März 1820, D-B 55 Nachl. 76,15 .

26 „Sketch of Music in London“, in: Quarterly Musical Review 2 (1820), S. 373-391, hier S. 384.

27 „Frankfurt am Mayn“, in: Allgemeine musikalische Zeitung 22 (1820), Sp. 856-863, hier Sp. 860.

28 Ebd. 


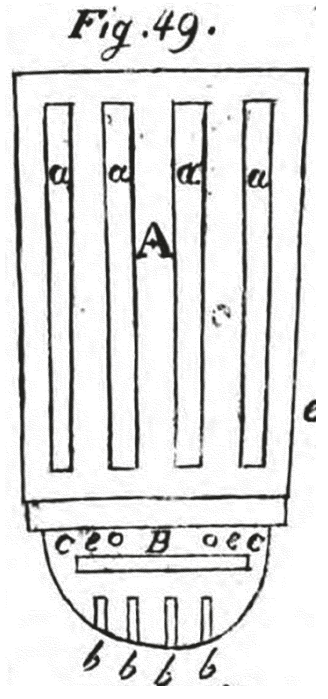

Abbildung 4: Saitenhalter nach Spohr bei Wettengel, Lehrbuch, Tafel VI.

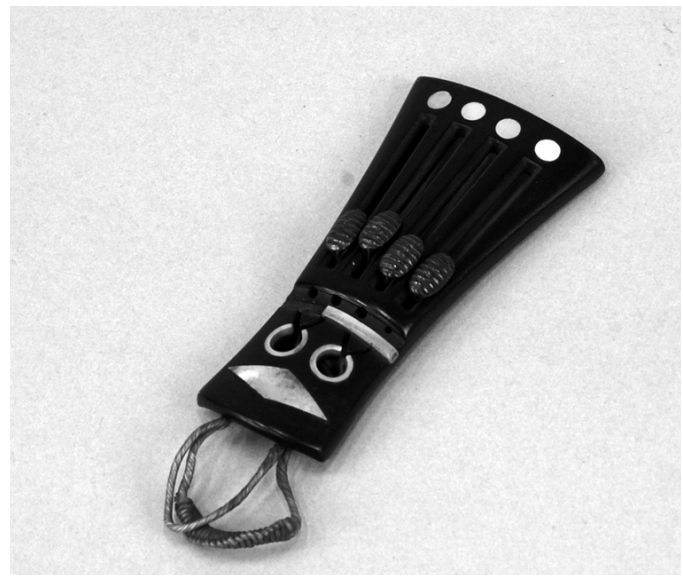

Abbildung 5: Saitenhalter nach Spohrs Beschreibung, DK-Km MMCCS No. D 74, Abdruck mit freundlicher Genehmigung durch The Danish Music Museum - Musikhistorisk Museum \& The Carl Claudius Collection.

fried Schramm, der während Spohrs dortigem Engagement auch als Orchesterdiener fungierte, ${ }^{29}$ kündigte an:

„Da der Herr Kapellmeister Spohr die Güte gehabt hat, mir ein Modell des von ihm verbesserten Saitenhalters mitzutheilen, so mache ich bekannt, dass von nun an solche Saitenhalter mit der nöthigen Anweisung wie sie auf der Violine befestigt werden, bey mir zu haben seyn werden. “30

Der Breslauer Instrumentenmacher Fichtel zog nach:

„Nach einer Probe des von Herrn Kapellmeister Spohr erfundenen Saiten-Halters, verfertige ich dergleichen, und kann versichern: daß jede Violine dadurch noch besser und vollkommener wird. Ein dergleichen Saiten-Halter kostet nebst einer dazu erforderlichen längeren G-Saite 1 Rtlr. 15 sgr. Cour. Für Viole und Cello läßt sich dann die nehmliche Verbesserung vornehmen. “31

Als Besonderheit des Saitenhalters empfinden die Zeitgenossen dabei immer noch den angeblich oder tatsächlich besseren Klang; den Vorteil durch die Vorrichtung für das Kinn erkennen sie wohl nicht. ${ }^{32}$ Zumindest spricht dafür die Darstellung des Spohr'schen Saitenhalters im umfangreichen Geigenbaukompendium von Gustav Adolph Wettengel. Er beschreibt zwar ausführlich die Beschaffenheit des Saitenhalters und Gefahren bei der Her-

29 Vgl. Spohr, Lebenserinnerungen, Bd. 1, S. 112f., sowie Göthel in: ebd., S. 345, Anm. 58; zu Schramms Biographie vgl. Klaus Grünke, Hans-Karl Schmidt und Wolfgang Zunterer, Deutsche Bogenmacher, Bubenreuth 2000, Bd. 1, S. 206.

30 G[ottfried] Schramm, „Anzeigen“, in: Intelligenzblatt zur allgemeinen musikalischen Zeitung 22 (1820), Sp. $23 f$.

31 Fichtel, „Für Violin-Freunde“, in: Neue Breslauer Zeitung (1821), S. 1410. - Bei ihm könnte es sich um den bei Lütgendorff angegebenen Gottlieb Fichtel handeln (Lütgendorff, Geigen- und Lautenmacher, S. 177 (6. Aufl. Bd. 2, S. 133)).

32 Zumindest im Falle des von Fichtel angebotenen Saiten-Halters für das Cello besteht dieser Vorteil freilich auch wirklich nicht. 
stellung, der Kinnhalter selbst fehlt jedoch sowohl im Text als auch in der beigefügten Zeichnung (vgl. Abb. 4). ${ }^{33}$ Immerhin deutet diese Zeichnung besser als Spohrs eigene Skizze an, wie hier die kürzeren Enden der Saite gestimmt werden sollen. Noch deutlicher zeigt ein im Danish Music Museum befindlicher „Saitenhalter [...] für Geige nach einem Modelle von Louis Spohr“34 die Stimm-Mechanik (vgl. Abb. 5). Darüber hinaus gibt Wettengel einen Grund an, warum wir heute Spohr zwar als Erfinder des Kinnhalters kennen, seine Weiterentwicklung des Saitenhalters jedoch aus unserem Bewusstsein entschwunden ist. Sie teilt mit ähnlichen Entwicklungen dieser Zeit ein Problem:

„Obgleich seit der Erfindung dieser Saitenhalter nur wenige Jahre verflossen sind, und sie oft wirklich mit Vortheil bei musikalischen Darstellungen gebraucht werden können, so sind sie doch schon beinahe ganz wieder aus der Mode gekommen, da sie der Haltbarkeit der Saiten entgegenwirken. “35

Im Zitat aus der Violinschule zu Beginn dieses Beitrags erwähnt Spohr, dass der Geigenhalter über dem Saitenhalter angebracht ist, nicht jedoch, dass die Saiten zwischen Steg und Saitenhalter ebenfalls abgestimmt werden sollen. Offensichtlich hat er also selbst auch diesen Gebrauch zwischen seiner Erfindung 1820 und dem Abschluss seiner Violinschule 1832 aufgegeben. Da Fichtel noch 1821 den Saitenhalter bewarb, Wettengel jedoch 1828 konstatierte, er sei aus der Mode gekommen, lässt sich der Zeitraum weiter eingrenzen. Im Umkehrschluss könnte Wettengels Feststellung freilich auch bedeuten, dass er zuvor in Mode gewesen sein muss. Gustav Schilling erwähnt ihn 1841 jedoch nur noch als Kuriosum:

Erinnere ich mich Recht, so versuchte der große Spohr einmal, durch Verkürzung des sogenannten Saitenhalters und Verbindung der Saiten in einem Bunde unter demselben der Violine eine höhere Vibrationskraft und dadurch vielleicht auch eine vollere Klangmasse zu verleihen; allein nicht blos, daß diese Veränderung gar keinen Einfluß auf die sonstige Construction und Produktionsfähigkeit des Instruments selbst äußerte, sondern sie kam auch nicht einmal zur allgemeinen Ausführung. ${ }^{36}$

Eine Instrumentenkunde von 1834 spricht zwar noch vom „Spohr'schen Saitenhalter“. Da hier jedoch von einem Abstimmen der Saiten nicht mehr die Rede ist, dafür aber von einem Teller ist, der das Halten des Instruments erleichtert, ist hier vermutlich bereits der Geigenhalter gemeint:

33 Gustav Adolph Wettengel, Vollständiges, theoretisch-praktisches auf Grundsätze der Akustik, Tonkunst und Mathematik, und auf die Erfahrungen der geschicktesten italienischen und deutschen Meister begründetes Lehrbuch der Anfertigung und Reparatur aller noch jetzt gebräuchlichen Gattungen von italienischen und deutschen Geigen [...], Ilmenau 1828, S. 138-141 und Tafel VI, Fig. 59-53, vgl. auch ebd. S. 142 und 350.

34 Hammerich, S. 100.

35 Ebd., S. 141. - In der Wortwahl wohl von dieser Quelle abhängig ist die Darstellung des Spohr'schen Saitenhalters in: Otto Bachmann, Theoretisch-praktisches Handbuch des Geigenbaues. Oder Anweisung, italienische und deutsche Violinen, Bratschen, Violoncello's, Violons, sowie Guitarren und Geigenbogen nach den neuesten Grundsätzen und in höchster Vollkommenheit zu verfertigen [...], Quedlinburg und Leipzig 1835, S. 25.

36 Gustav Schilling, Geschichte der heutigen oder modernen Musik in ihrem Zusammenhange mit der allgemeinen Welt- und Völkergeschichte, Karlsruhe 1841, S. 752. 
Der Capellm. Spohr [...] erfand eine Art Saitenhalter, welcher unten wie ein kleiner Teller geformt ist, und sich beim Spielen besser an den Backen anschließt, und gewöhnlich auch nur Spohr'scher Saitenhalter genannt wird. ${ }^{37}$

Dies führt zu der Frage, wann Spohr den Eigenwert des Geigenhalters erkannte. Er selbst spricht von seinen „zahlreichen Schülern“, bei denen sich sein Nutzen bewährt habe. Nun haben wir bereits zwei Schüler kennengelernt, die den Geigenhalter wohl nicht verwendeten: Ferdinand David und Hubert Ries erwähnen ihn in ihren eigenen Violinschulen nicht. David und Ries studierten beide ab 1823 bei Spohr. ${ }^{38}$ Dies könnte ein Hinweis sein, dass der Geigenhalter zu dieser Zeit noch nicht so weit entwickelt war, wie ein Jahrzehnt später in Spohrs Violinschule dargestellt, und daher vielleicht auch noch nicht bei seinen Schülern im Gebrauch war. Die bisher einzige Quelle zu einem deutschen Spohr-Schüler, der einen Geigenhalter verwendete, ist ein Tagebucheintrag von Carl Bargheer vom 17. August 1849:

„Ich ging des Morgens mit H. Schultz zum Instrumentenmacher Schonger, um einen Teller zu der Guarneri zu bestellen; [... “39

Um diese Zeit gibt es auch bereits Vorschläge für Alternativen zum Geigenhalter:

„Der Spohr'sche Violinhalter (Teller) ist zwar von manchem Violinisten für zweckentsprechend anerkannt worden, dagegen ist es unleugbar, dass Wenige sich zu diesem Halter haben gewöhnen können, weshalb Unterzeichneter [...] auf die Idee gekommen ist, einen leichten 1/4 Zoll breiten, mit einer Plasance-Schnalle versehenen Riemens sich zu bedienen, welcher unter dem Saitenhalter durchgezogen und um den Hals befestigt wird. Hieraus erwachsen nachstehende Vorteile:

1) liegt das Instrument fest am Halse, unter dem Kinn ohne dass es nur den Steg berührt, gedrückt und in Folge grosser Anstrengungen mit Schweiss befeuchtet wird,

2) gewinnt das Instrument dadurch einen hellen und bei Weitem stärkeren Ton, weil es nicht gedrückt und gedeckt wird,

3) werden Halsbinden geschont und nicht im Mindesten derangirt, weil der Kopf ein und dieselbe Lage behält, und bei den schwierigsten Passagen in der Applicatur, die sonst unentbehrliche Hülfe und Haltung des Kinns hier gänzlich wegfällt,

4) habe ich die Erfahrung gemacht, dass mit Hülfe des fraglichen Violinhalters 6-8 Stunden die schwierigsten Compositionen vorgetragen worden sind, ohne dabei die geringste Ermüdung, was doch gewöhnlich geschieht, verspürt zu haben. “ 40

Dafür experimentierten durchaus einige Musiker mit dem erwähnten Saitenhalter. Spohr erwähnt in seinem Bericht „die Londoner Geigenmacher“, also mehrere Instrumentenbauer, welche diese Saitenhalter anfertigten. Und eine englische Übersetzung der Violinschule

37 Wilhelm Schneider, Historisch-technische Beschreibung der musicalischen Instrumente, ihres Alters, Tonumfang und Baues, ihrer Erfinder und Verbesserer, Virtuosen und Schulen, nebst einer faßlichen Anweisung zur gründlichen Kenntnis und Behandlung derselben, Neiße und Leipzig 1834, S. 68ff., Anm. **; fast wörtlich übernommen in: Art. „Geige“, in: Musicalisches Conservations-Lexikon. Enzyklopädie der gesammten Musik-Wissenschaft, hrsg. von August Gathy, ${ }^{2} 1840$, S. 160.

38 Vgl. C. B., „Verzeichnis der Schüler von Louis Spohr“, in: Niederrheinische Musik-Zeitung 7 (1859), S. $150 \mathrm{ff} .$, hier S. 150.

39 Carl Bargheer, Tagebuchnotizen 27. August 1848 bis November 1849, D-Dt Mus-h 1 B 36, Bl. 8v, zit. n. ders., Fidellieder für Bariton, Violine und Klavier nach Texten von Theodor Storm. Digitale Edition des Werkes plus Zusatzinformationen rund um Werk und beteiligte Personen, hrsg. von Joachim Veit u. a., Detmold 2012, <http://www.edirom.de/llb-bargheer-edition/index.html> (eingesehen 25.01.2015).

40 [Ignaz von] Leibitz-Piwinicki, „Ein Vorschlag um die Geige und Bratsche an dem Halse des Tonkünstlers in der Art zu befestigen, dass die Griffe in der Applicatur ohne Mühe, Anstrengung und mit Präcision gehandhabt werden können, wobei das Instrument fortwährend in derselben Lage bleibt", in: Berliner musikalische Zeitung 3 (1846), H. 26, dort auf der vierten unpaginierten Seite. - Eine Ent- 
verweist auch auf die Rezeption des Geigenhalters bei einem Londoner Instrumentenbauer, dessen Modell im Gegensatz zu Spohrs Konstruktion unabhängig vom Saitenhalter an der Geige befestigt wird:

"Though this contrivance has long proved its efficacy, the Violin-holder invented by Dr. J. Steward, (to be obtained at Messrs. Wither's, 31, Conventry Street, London,) may claim a preference, possessing, as it does, all the advantages of the above invention, with this in addition, that it raises the Violin close up to the chin of the player, and is most readily and simply attached to the instrument. This holder consists of two narrow blocks of wood, placed opposite each other, above and below the Violin, and clamped to the instrument immediately on the left of the tail-piece by a screw-pin. In using it, the chin of the player should be laid upon the tail-piece, which, together with the upper block of the holder, affords a firm purchase of the Violin, without any kind of contact detrimental to its free vibration. - ED. “41

Mit dieser Quelle sind wir zwar bereits in den 1870er Jahren, den Geigenhalter von Stewart finden wir jedoch schon in früheren Texten, wie 1846 in einer Anzeige von "J. Stewart's registered violin and tenor holder" in der Musical World. Die halbseitige Anzeige enthält neben den Empfehlungen anderer führender Londoner Geiger dieser Zeit auch ein paar Zeilen des Spohr-Schülers Henry Blagrove: „I have to congratulate you on a most simple, useful, and effectual invention for holding the violin, and consider it a great boon to amateurs and professors of that instrument. "42 Hieraus lässt sich vermutlich schließen, dass Blagrove spätestens zu diesem Zeitpunkt eine Kinnstütze verwendete. Da er seinen Unterricht bei Spohr 1833 begann, als Spohrs Violinschule gerade erschienen war, lernte er jedoch wahrscheinlich schon in Kassel die Spohr'sche Kinnstütze kennen. ${ }^{43}$ Eine Besprechung von Stewarts Geigenhalter nimmt - im Gegensatz zum oben zitierten Kommentar aus einer englischen Übersetzung der Violinschule - keinen Bezug auf Spohr. Dieser Text stellt die „utility“ durch leichte Anbringung und Transport heraus, jedoch nicht mehr den grundsätzlichen Nutzen eines Geigenhalters. Dies könnte darauf deuten, dass sich eine oder mehrere Formen des Geigenhalters zu dieser Zeit in England durchgesetzt haben. Dagegen spricht allerdings der Hinweis, dass gerade Anfänger durch den Geigenhalter einen schnelleren Fortschritt machen:

„Stewart's Violin Holder. - The utility of this simple and highly efficient apparatus, for facilitating the holding of the Violin, has been acknowledged by all professors of that instrument, and is now in course of being universally adopted. The holder may be carried in the waistcoast pocket, and attached to the violin instaneously, when required. We ourselves have tested the use of the instrument, and are warranted in strongly recommending so admirable and efficient a contrivance. To the beginner, above all others, it will prove of the greatest service in facilitating his progress, and rendering compassable, in a brief period, difficulties which at present, can only be surpassed by a long course of practice. Mr. Stewart, has conferred a boon, on all violin players by his ingenious invention. " ${ }^{4}$

In den Folgejahren rückte der Geigenhalter offensichtlich von der von Spohr bevorzugten Anbringung über dem Saitenhalter auf Position seitlich des Saitenhalters, so wie wir es heute kennen. Zumindest soll der Vorteil eines 1884 patentierten Geigenhalters darin bestehen, wieder wie von Spohr gefordert, über dem Saitenhalter angebracht zu sein:

gegnung auf diesen Vorschlag hält die hier angeführten Gründe für „,nicht gerade stichhaltig“ („Kleine Zeitung", in: Neue Zeitschrift für Musik 25 [1846], S. 62).

41 Henry Holmes in: Spohr's Violin School, hrsg. von Henry Holmes, London [1878], S. 4. Datierung nach Folker Göthel, Thematisch-Bibliographisches Verzeichnis der Werke von Louis Spohr, Tutzing 1981, S. 316.

42 „J. Stewart's registered violin and tenor holder", in: Musical World 21 (1846), S. 663.

43 Zum Datum von Blagroves Unterricht bei Spohr vgl. C. B., „Verzeichnis“, S. 150

44 „Stewart's Violin Holder“, in: Musical World 22 (1847), S. 25 f. 
„Violinists will be glad to learn that a new chin holder has been invented which posesses many advantages for the player. It is appropriately called the 'Spohr,' its principal feature being that it can be fixed immediately over the tail-piece of the instrument, which position Spohr advocated as the correct place for fitting the violin holder. In fact, so important did this authority think of this central hold on the instrument, that in his work on the violin he wrote a long paragraph of this subject $[\ldots]^{\text {« } 45}$

Ein Hinweis von Walter Kolneder deutet auf eine Verbreitung der Kinnstütze im Frankreich der 1850er Jahre:

„Bériot beschrieb in seiner Violinschule (1858) den Kinnhalter als eine aus Deutschland kommende Neuerung. Seit Spohr sind unzählige Modelle geschaffen worden und kürzere oder längere Zeit über in Gebrauch gewesen. “46

Allerdings findet sich in Bériots Méthode de violon weder ein Hinweis auf die Kinnstütze selbst noch gar auf eine deutsche Herkunft. ${ }^{47}$ Stattdessen schlägt Bériot vor, die Geige über dem Kragen festzuklemmen oder - wie bei Ferdinand David - ein Tuch oder Kissen unterzulegen:

„Le Violon placé sur la clavicule gauche, appuyé contre le con, et soutenu par le col de l'habit et du gilet qui le font incliner naturellement vers la droite. Les entfants qui par leurs vetements légers sont privés de l'appui du col peuvent le remplacer par un mouchoir ou un coussinet afin d'éviter l'habitude disgracieuse de lever le paule pour soutenir l'instrument. “48

Woher Kolneders Information stammt, lässt sich derzeit nicht nachvollziehen. Gleiches gilt für die etwas kryptische Formulierung in einem Artikel über verschiedene Geigenschulen:

Spohr appelle ainsi la mentionnère destinée à favoriser la tenue du violon qui glisse facilement sous le menton dans le démanchés. Les violinistes Bériot, Sivori et Léonard ont adopté chacun une mentionnère différente. ${ }^{49}$

Dieser Autor schreibt Bériot die Entwicklung einer eigenen Kinnstütze zu, für die sich zumindest in seiner Méthode kein Beleg findet. Vermutlich meint der Autor hier das vorgeschlagene Kissen zwischen Schulter und Geige. Dass Spohr wiederum den Anspruch angemeldet hatte, Erfinder der Kinnstütze zu sein, konnten französische Leser zumindest wissen, wenn sie die französische Übersetzung seiner Violinschule in die Hand nahmen; diese Übersetzung zitiert den Geigenhalter sogar im Titel: École ou Méthode Pour le Violon à l'aide du Teneur de Violon. ${ }^{50}$ Im Gegensatz zu Deutschland und Großbritannien war Spohr

45 „The Patent 'Spohr' Chin Holder“, in: Musical Standard 27 (1884), S. 258.

46 Walter Kolneder, Das Buch der Violine, Zürich 1972, S. 43; fast wörtlich übernommen in: Cornelia Büning, „Kinnhalter, Kinnstütze“, in: Lexikon der Violine. Baugeschichte-Spielpraxis-Komponisten und ihre Werke - Interpreten, hrsg. von Stefan Drees, Laaber 2003, S. 344f., hier S. 344.

47 C[harles] de Bériot, Méthode de Violon, Paris 1857; darüber hinaus war mir ein späterer französischenglischer Druck zugänglich: Ch[arles] de Bériot, Méthode de Violon, Mainz o. J., Pl.-Nr. 21873. Roland Folter hat für mich dankenswerterweise in seinem Exemplar des Drucks Mainz, Pl.-Nr. 15000 nachgesehen sowie in einer späteren deutsch-französischen Ausgabe Mainz [1898], Pl-Nr. 26337. In keiner dieser Ausgaben findet sich ein Hinweis auf die Kinnstütze.

48 Bériot, Méthode, S. 4.

49 Félix Huet, „Étude sur les différentes écoles de violon depuis Corelli jusqu’à Baillot. Précédée d'un examen sur l'art de jouer des instruments à archet au XVIII siècle", in: Mémoires de la société d'agriculture, commerce, science et arts du département de la Marne (1878-1879), Chalons-sur-Marne 1880, S. 275-420, hier S. 345, Anm. 1.

50 Louis Spohr, École ou Méthode Pour le Violon à l'aide du Teneur de Violon, übers. von Heller, Paris [1838] (Datierung nach Göthel, Thematisch-Bibliographisches Verzeichnis, S. 316). 
in Frankreich allerdings nie populär. ${ }^{51}$ Das Wissen um Spohr verblasste, und ein französischer Lexikonartikel aus den 1920er Jahren verweist nur auf die Möglichkeit, Spohr sei der Erfinder gewesen:

„On ignore le pays d'origine de la mentionnère, et le nom de son inventeur. Il est possible qu'elle vienne d'Allemagne; lorsque Spohr, le célèbre violonist et compositeur, vint à Paris en 1819, il en avait a une à son violon, et aucun violoniste français ne s'en servait à cette époche. "52

Die Begründung für Spohrs mögliche Erfindung bezieht sich dann auch nicht auf die Violinschule, sondern auf Spohrs Paris-Aufenthalt 1820, der hier fälschlich mit 1819 angegeben ist. ${ }^{53}$ Wegen dieser Ungenauigkeiten taugt dieser Text auch nicht als Beleg dafür, dass Spohr bei seinem Paris-Aufenthalt bereits mit Kinnstütze gespielt habe.

Offensichtlich entwickelte Spohr seine Kinnstütze aus seiner heute vergessenen Weiterentwicklung des Saitenhalters. Während Spohrs ab 1823 unterrichtete Schüler Ferdinand David und Hubert Ries die Kinnstütze wohl nicht verwendeten, setzt Spohr in seiner Violinschule 1833 die Verwendung der Kinnstütze bei „zahlreichen Schülern und vielen anderen Geigern" voraus, vermutlich vorrangig Geiger aus seinem näheren Umfeld. Zu Beginn des 20. Jahrhunderts scheint die Kinnstütze etabliert gewesen zu sein. Wie die Verbreitung sich jedoch im Detail gestaltete, wird sich erst durch die Erschließung weiterer Quellen zeigen lassen.

51 Zur Spohr-Rezeption in Frankreich vgl. Hélène Cao, Louis Spohr ou Le don dêtre heureux, Paris 2006, S. 64-67 and 114-115; Clive Brown, Louis Spohr, S. 260 (dt. Übersetzung S. 310f.; diese deutsche Übersetzung hat dem englischen Original gegenüber den Vorteil, dass hier die französischen Originalquellen belegt sind und nicht die Übersetzungen in englischen Periodika).

52 Art. „Le mentionnère“, in: Encyclopédie de la musique et dictionnaire du conservatoire, Teil 2 Technique, esthétique, pédagogie, Bd. 3 Technique instrumentale, Paris 1927, S. 1740.

53 Vgl. Louis Spohr, „Briefe aus Paris“, in: Allgemeine Musikalische Zeitung 23 (1821), Sp. 139-144, 156162, 177-184 und 189-195. 